\title{
Keeping CRISPR/Cas on-Target
}

\author{
Muhammad Jamal', Faheem Ahmed Khan ${ }^{2}$, Lin $\mathrm{Da}^{1}$, \\ Zeshan Habib ${ }^{1}$, Jinxia Dai ${ }^{1,2 *}$ and Gang Cao ${ }^{1,2 *}$ \\ ${ }^{1}$ State Key Laboratory of Agriculture Microbiology, Huazhong \\ Agricultural University, Wuhan, Hubei, 430070, P. R. China \\ ${ }^{2}$ College of Animal Science and Medicine, Huazhong \\ Agricultural University, Wuhan, Hubei, 430070, P.R. China. \\ *Corresponding author: jxdai@mail.hzau.edu.cn; \\ gcao@mail.hzau.edu.cn
}

DOI: http://dx.doi.org/10.21775/cimb.020.001

Abstract

CRISPR/Cas, a microbial adaptive immune system, has recently been reshaped as a versatile genome editing approach, endowing genome engineering with high efficiency and robustness. The DNA endonuclease Cas, a component of CRISPR system, is directed to specific target within genomes by guide RNA (gRNA) and performs gene editing function. However, the system is still in its infancy and facing enormous challenges such as off-target mutation. Lots of attempts have been made to overcome such off-targeting and proven to be effective. In this review we focused on recent progress of increasing the CRISPR specificity realized by rational design of gRNA and modification of Cas9 endonuclease. Meanwhile the methods to screen off-target mutation and their effects are also discussed. Comprehensive consideration and rational design to reduce off-target mutation and selection of effective screening assay will greatly facilitate to achieve successful CRISPR/Cas system mediated gene editing.

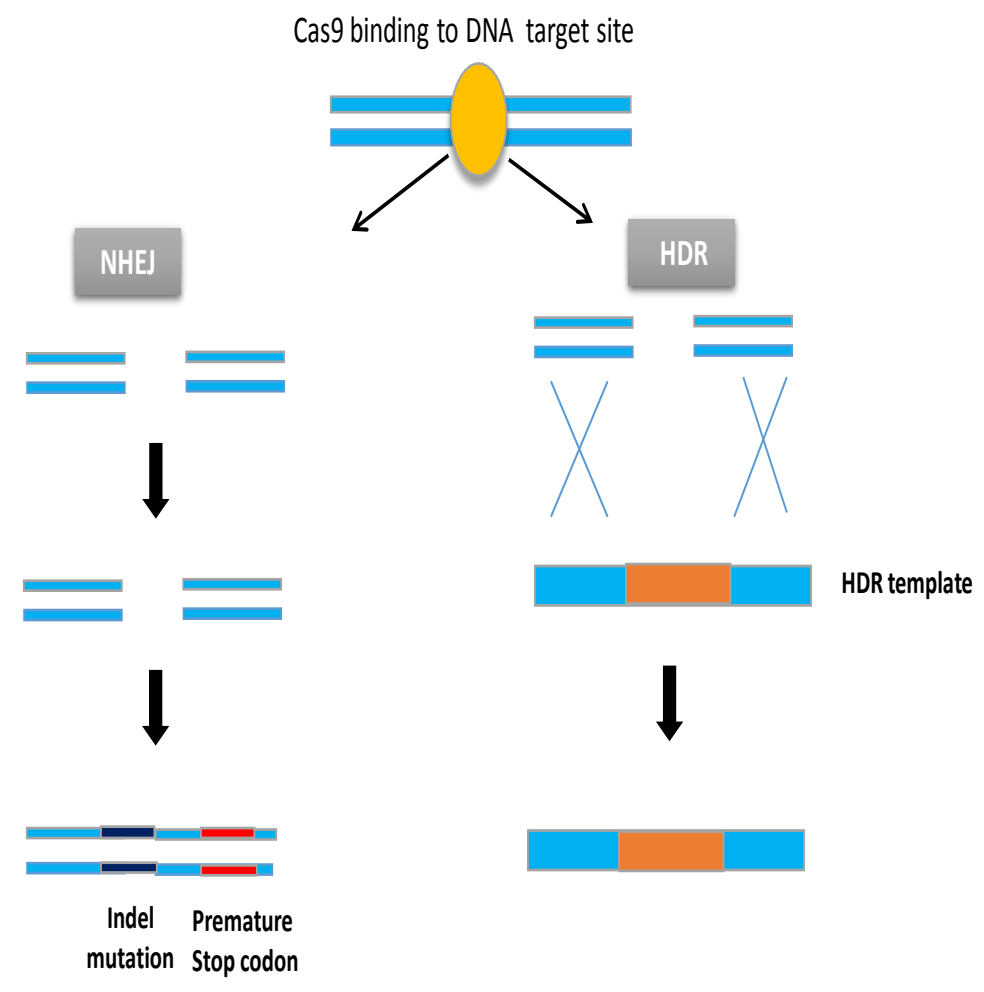

Gene knock out

Gene knock in

Figure 1. Pathway for DSB repair induced by CRISPR. Left: Non-homologous end joining (NHEJ), the ends of a DSB are processed by endogenous DNA repair machinery and rejoined, which can result in random indel mutations possibaly resulting in gene knockout. Right: homology directed recombination. A repair template in the form of a plasmid or single stranded oligodeoxyribonulceotides (sSODN) can be supplied to leverage the HDR pathway, which allows high fidelity and precise editing. Single-stranded nicks to the DNA can also induce HDR. 


\section{Introduction}

CRISPR (clustered, regularly interspaced, short palindromic repeat)/Cas (CRISPR-associated protein) system was first discovered in 1987 when researchers found a set of 29 nucleotide(nt) repeats downstream of the iap gene (Ishino et al., 1987). In Bacteria and Archea, CRISPR/Cas is a defensive mechanism to direct degradation of foreign genome by means of CrRNA (CRISPR RNAs) complementary to sequences within invading viral genome or plasmid DNA (Bhaya et al., 2011; Terns and Terns, 2011; Wiedenheft et al., 2012). It has been shown that $44 \%$ of a representative set of 703 archaeal possess one or more CRISPR/Cas modules in the genome(Deveau et al., 2010; Koonin and Makarova, 2009). Recently, tremendous data demonstrated this system has the capability to manipulate the genome of almost all organisms including bacteria, yeast, fruit flies, zebrafish as well as mice (shown in Table 1).

The Streptococcus pyogens's Type II CRISPR system consist of precursor-CRISPR RNA (pre-crRNA), transactivating crRNA (tracrRNA) and CRISPR associated protein 9 (Cas9). The pre-crRNA is the primarily transcribed product of spacers and repeats in the CRISPR array. After cleavage of the pre-crRNA by RNase III, the mature crRNA (known as gRNA) containing sequence complementary to target site is generated to hybridize with tracrRNA and finally form a complex with Cas9 to exert target DNA editing (Horvath and Barrangou, 2010). Thus, target cleavage requires the crRNA, the tracrRNA and Cas9 to work coordinately. Two crucial domains of Cas9, the RuvC and $\mathrm{HNH}$ domains have been found to be pivotal for Cas9 function. Each of the two Cas 9 domains cleaves one DNA strand of the protospacer region, 3-4 nt upstream of the recognition motif protospacer-adjacent motif (PAM)(Jinek et al., 2012). In the Streptococcus pyogenes type II CRISPR system, it has been recently suggested that crRNA fused to tracrRNA is sufficient to direct Cas9 protein to mediate sequence-specific cleavage, allowing a programmable genome editing (Jinek et al., 2012).

Although the CRISPR/Cas mediated genome editing is robust and efficient, the technique still requires optimization. Sequence features control the specificity of CRISPR/Cas mediated gene editing. The gRNA/Cas9 complex tolerates 1-3 or even more mismatches in their targets, as Cas9 was shown to bind to many sites in human genome other than the intended targets. Thus, genomic sequences with similarity to target site would be more vulnerable to be mutated. Several groups independently reported that Cas 9 can cause unwanted mutations at off-target sites that differ by up to $5 \mathrm{nt}$ from the on-target sites in human and several other organisms (Fujii et al., 2013; Li et al., 2013; Shan et al., 2013). For some sites, the rate of mutation at off-target sites could be as high as, or even higher than, that of target site (Fu et al., 2013).Off-targets are of paramount importance to be considered during genome engineering processes because off-target cleavage followed by erroneous repair pathway could give rise to mutations at an undesired locus. Similarly, chromosomal rearrangements as a result of offtargeting can cause malignancies due to activation of oncogenes or suppression of tumor-suppressor gene function.

Off-target mutations can be caused both by Cas 9 and gRNA. Therefore, improvement of the current generation of CRISPR/Cas platform is mostly focused on these two factors. Several efforts aiming to improve CRISPR specificity should be performed prior to using CRISPR/ Cas9 mediated genome editing, including the rational design of gRNA, selection of eligible CRISPR nuclease, choice of suitable target sites and delivery of Cas9-sgRNA into cells followed by rapid degradation of the nuclease. Here we reviewed the recent advances in these approaches to reduce the off-target mutation rate.

\section{Judicious gRNA design}

In order to obtain the efficient on-target editing, it is foremost important to consider the composition and structure of gRNA as they will affect the off-target mutations rates (Cho et al., 2014). Recent studies in mammalian cells showed that Cas9 can tolerate several nucleotide mismatches between a DNA-RNA hybrid in a sequence dependent manner based on the quantity, position, and distribution of mismatches (Fu et al., 2014; Horvath and Barrangou, 2010; Ran et al., 2013). Usually a gRNA with fewer than three mismatches to a secondary genomic target site is preferred to induce the off-target effects (Cong et al., 2013; Mali et al., 2013a). Even mismatches up to five base pairs are tolerated by Cas9 in the PAM distal region (Cong et al., 2013; Fu et al., 2013; Mali et al., 2013b). Therefore, gRNA needs to be carefully designed to avoid off-target effects, especially those with mismatches to the 2 bases proximal to PAM sequence(Cradick et al., 2013). The important features for gRNA design are discussed immediately below.

\section{i. Protospacer adjacent motif (PAM)}

The PAM recognized by one of the Cas 9 domains is strictly required to be immediately next to the $3^{\prime}$ end of the target sequence. The binding of Cas 9 to PAM is required for opening and cleaving of the target site (Nishimasu et al., 2014). In S. pyogenes, the PAM site is repeated every 8 nucleotide on average. There are different types of PAM sequence for different bacterial species (Table 2). In order to design a sgRNA, the off-target sites should have a limited sequence homology with on-target sites and absence of PAM with either 5'-NGG or 5'-NAG sequences. Cas9 prefer to bind to NGG PAM sites, with GGG being the sequence most frequently bound (Kuscu et al., 2014).

\section{ii. PAM distal vs PAM proximal nucleotides}

For a 20 nt gRNA, the sequence of 5-12 nt closest to PAM, known as the seed sequence, are most critical for the binding specificity. Mismatches in the PAM-distal nucleotides show less effect on disturbing the sgRNAtarget DNA hybrid than those in the PAM-proximal nucleotides (PAMPNs). Therefore, it suggests that PAMproximal nucleotides are the major determinants for Cas9 binding to the target sites (Cong et al., 2013; Fu et al., 2013; Jinek et al., 2012; Kuscu et al., 2014; Mali et al., 2013b).Chromatin immunoprecipitation followed by sequencing (ChIP-seq) suggested that only ten base pairs 
Table 1. Organisms modified by CRISPR Cas system.

\begin{tabular}{|c|c|c|}
\hline Groups & Organism & References \\
\hline Vertebrates & $\begin{array}{l}\text { Axolot } \\
\text { Frog } \\
\text { Human } \\
\text { Monkey } \\
\text { Mouse } \\
\text { Pig } \\
\text { Zebrafish } \\
\text { Rabbit }\end{array}$ & $\begin{array}{l}\text { (Flowers et al., 2014) } \\
\text { (Blitz et al., 2013; Guo et al., 2014) } \\
\text { For review see (Sander and Joung, 2014a) } \\
\text { (Niu et al., 2014) } \\
\text { (Sander and Joung, 2014a) } \\
\text { (Hai et al., 2014) } \\
\text { (Auer et al., 2014) } \\
\text { (Yang et al., 2014) }\end{array}$ \\
\hline Invertebrates & $\begin{array}{l}\text { Freshwater flea } \\
\text { Fruit fly } \\
\text { Round worm } \\
\text { Silkworm }\end{array}$ & $\begin{array}{l}\text { (Hai et al., 2014) } \\
\text { (Gratz et al., 2013) } \\
\text { (Waaijers and Boxem, 2014) } \\
\text { (Daimon et al., 2014; Wang et al., 2013b) }\end{array}$ \\
\hline $\begin{array}{l}\text { Plants } \\
\end{array}$ & $\begin{array}{l}\text { Corn } \\
\text { Liverwort } \\
\text { Rice } \\
\text { Thale cress } \\
\text { Tobacco } \\
\text { Wheat } \\
\text { Sorghum } \\
\text { Sweet orange }\end{array}$ & $\begin{array}{l}\text { (Liang et al., 2014) } \\
\text { (Sugano et al., 2014) } \\
\text { (Belhaj et al., 2013) } \\
\text { (Belhaj et al., 2013) } \\
\text { (Belhaj et al., 2013) } \\
\text { (Upadhyay et al., 2013) } \\
\text { (Jiang et al., 2013c) } \\
\text { (Jia and Wang, 2014) }\end{array}$ \\
\hline Bacteria & $\begin{array}{l}\text { Streptococcus } \\
\text { pneumoniae } \\
\text { And E.coli } \\
\text { Lactobacillus reuteri }\end{array}$ & $\begin{array}{l}\text { (Jiang et al., 2013b) } \\
\text { (Oh and van Pijkeren, 2014) }\end{array}$ \\
\hline
\end{tabular}

in the PAM proximal regions are sufficient to mediate the Cas9 binding (Kuscu et al., 2014).

\section{iii. GC content of sgRNA}

The GC content of sgRNA can also affect off-target effects. High GC content gives more stability to RNA-DNA hybrid (Sugimoto et al., 1995). High rates of mutagenesis have been observed for off-target sites when using gRNA with only 30\% GC content (Fu et al., 2013; Pattanayak et al., 2013).In mammalian cells, sgRNA with medium GC content is more vulnerable to off-target mutation compared to those with high or low GC content (Wang et al., 2014). Another study showed that a high or a low number of GC in sgRNA showed a reduced mutagenic activity (Doench et al., 2014). It's very likely that Cas9/sgRNA efficiency at on-target and 
Table 2. Types of PAM sequence in different bacteria.

\begin{tabular}{|c|c|c|}
\hline Bacterial species & PAM sequence & References \\
\hline $\begin{array}{l}\text { Streptococcus pyogens } \\
(\mathrm{Sp})\end{array}$ & NGG & (Jinek et al., 2012) \\
\hline S. solfataricus I-A1 & $\mathrm{CCN}$ & (Manica et al., 2011) \\
\hline S. solfataricus I-A2 & TCN & $\begin{array}{l}\text { (Gudbergsdottir et al., 2011; } \\
\text { Lillestøl et al., 2009) }\end{array}$ \\
\hline Neisseria meningitidis $(\mathrm{Nm})$ & NNNNGATT & (Hou et al., 2013) \\
\hline $\begin{array}{l}\text { Streptococcus thermophiles } \\
\text { (St) Type II }\end{array}$ & $\begin{array}{l}\text { NGGNG } \\
\text { NNAGAAW }\end{array}$ & $\begin{array}{l}\text { (Deveau et al., 2008; Horvath and } \\
\text { Barrangou, 2010) }\end{array}$ \\
\hline $\begin{array}{l}\text { Streptococcus mutan } \\
(\mathrm{Sm})\end{array}$ & NGG or NAAR & (van der Ploeg, 2009) \\
\hline
\end{tabular}

off-target sites is governed by the thermodynamic stability of the Watson-Crick base pairing.

\section{iv. Epigenetics and chromatin structure}

Understanding the relationship between epigenetics and Cas9/dCas9 (mutant form of Cas9) targeting is important to reduce the off-target mutagenesis effects. Chromatin structure plays an important role in dCas 9 binding as most of the binding site for dCas 9 were found to be present in open chromatin region (promoter, 5'UTR, exons) (Kuscu et al., 2014).This is further supported by a research showing that Cas 9 binding is affected by the chromatin structure due to compaction(Sander and Joung, 2014). However, it is reported that $\mathrm{CpG}$ methylation does not affect DNA cleavage in vitro and Cas 9 could introduce indels in a high methylated regions in vivo (Hsu et al., 2013; Perez-Pinera

\section{Modification of gRNA}

A

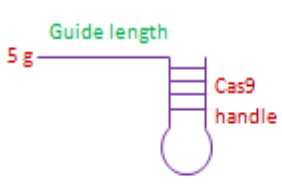

standard gRNA

(20 nucleotides)

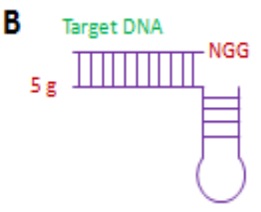

truncated gRNA

(17 nucleotides)

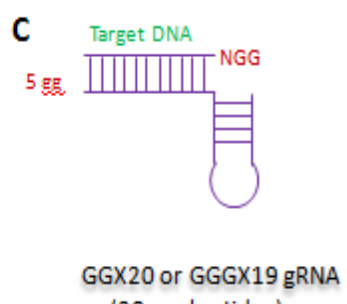

(20 nucleotides)

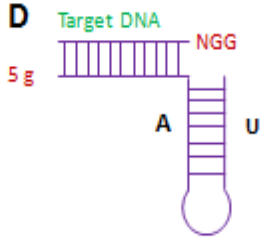

gRNA with $\mathrm{A}-\mathrm{U}$ base pair flipping with extensive base pairs in hairpin'

(20 nucleotides)

\section{Modification of Cas 9}

E

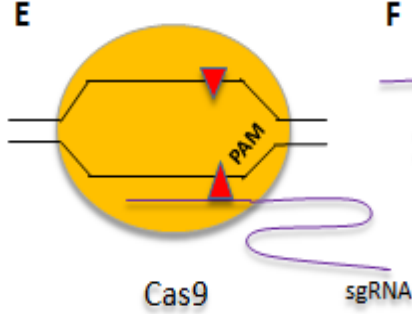

$\mathbf{F}$

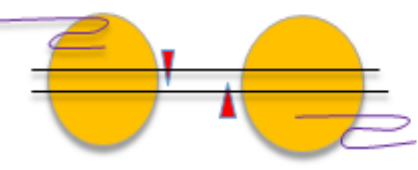

Cas9n
G

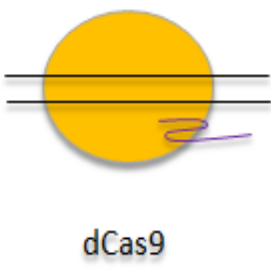

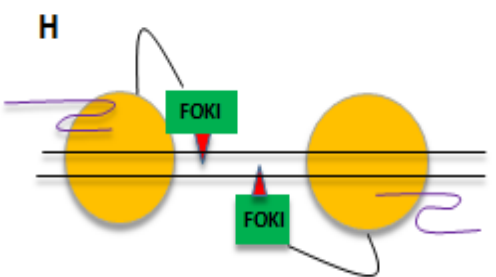

dCas9-FOKI

Figure 2. Schematic strategies for reducing off-target mutations. Four different forms of gRNAs: A. showing standard gRNA of normal length. B. gRNAs with truncated nuclecutide sequence $17 \mathrm{nts}$. C. gRNA having two extra guanines ( $g$ X20) or gRNAs (gggX19). D. gRNA with A-U base pair flipping and stem extension enhance the specificity of RNA-guided genome editing, compared to conventional normal sgRNAs. E. normal Cas9 version. F. Cas 9 nickase guided by two gRNAs cutting double strand of DNA. G. dCas9, double mutant Cas9 with DNA binding ability but lacking cleaving ability. H. dCas9 fusion with FOKI nuclease cutting double strand of DNA with high efficiency. 
et al., 2013). It is of vital importance to evaluate Cas9 binding and cleavage of genomic loci in cells with different chromatin states.

\section{v. modified gRNAs scaffold}

The truncated gRNAs (tru-gRNAs) with shorter sequence ( $<20$ nucleotides, usually 17 or 18 ) can effectively improve the specificity of Cas9 (Fu et al., 2014) (shown in Figure 2B). It is reported that successful human genome editing was achieved by tru-gRNAs(Fu et al., 2013; Pattanayak et al., 2013). The off-target mutagenesis was largely reduced about 5000-fold by using tru-gRNA (Fu et al., 2014). Intriguingly, truncation at the 5'end of gRNA increase the on-target specificity, while truncation at the $3^{\prime}$ end of gRNA reduces the on-target cleavage activity of cas 9 nucleases (Fu et al., 2013; Hwang et al., 2013). Moreover, truncation of the standard gRNA by 1-3 nucleotides showed comparable activity to that of the full length gRNA. Notably, truncation of gRNA cannot be more than 3 nucleotides (16 nucleotides gRNA) as it will lost the locus targeting activity(Fu et al., 2014). Genome wide analyses revealed that tru-gRNAs possess less off-target double stranded breaks. Recently tru-RFNs (combination of tru-gRNA pair with RNA guided FOKI nucleases) showed high activity in human cancer cell lines and embryonic stem cells with reduced undesirable off-target effects (Wyvekens et al., 2015). The tru-gRNA provides Cas 9 with the necessary energy for binding to target site and reduces the affinity to off-target site. Usually, the full length gRNA provides more energy than the required, allowing the Cas9 to bind to unspecific sequences. In addition, the efficiency of trugRNA can be further enhanced when truncated gRNAs are conjugated with paired Cas9 nickases (Fu et al., 2014). Furthermore, the gRNA containing additional guanine bases at the $5^{\prime}$ end ( $5^{\prime}$-GGX20 or 5'-GGGX19) makes it more specific to the target by an unknown mechanism, perhaps by changing the gRNA stability, concentration or secondary structure (Cho et al., 2014; Kim et al., 2015) (shown in Figure 2C). In another strategy the introduction of an A-U base pair to the gRNA scaffold, stabilizes the gRNA scaffold. Besides, extension of the base pair in the hairpin increases the binding rate of gRNA to target site and finally enhances its on-target specificity (Chen et al., 2013) (shown in Figure 2D).

\section{Web based designing of $g R N A$}

Experimental identification of off-target sites by in vitro or in vivo methods is time consuming. Therefore, in silico prediction tools are broadly applied. The first generations in silico tools were mostly designed according to the degree of similarity between the target site and secondary sites in the genome. The recently developed tools predict unique genomic sites with minimum sequence homology to the secondary genomic sites (Heigwer et al., 2014; Sander et al., 2010)(shown in Table 3). Moreover these tools also provide the sequence of oligonucleotides for gRNA construction with paired Cas9n and RNA guided FOKI nucleases. (Sander et al., 2010).

The use of multiple tools at a time during designing process can be advantageous as every tool has its own features. Although these tools have contributed to Cas 9 specificity yet there are some factors such as seed sequence abundance, target sequence abundance and epigenetic state, need to be further considered.

\section{Selection of eligible Cas9}

RNA guided endonucleases(RGENs) including Cas9 are regarded as an important member of engineered nucleases due to their genome editing capabilities (Kim et al., 2014). They act on genome and cleave it by producing double stranded breaks (DSBs) at specific sites which are repaired by the cell endogenous DNA repair machinery(Carroll, 2014). There are three variants of Cas 9 so far to be used for genome engineering.

Firstly, the wild type Cas9 with both RuvC and $\mathrm{HNH}$ domains cleave double strand DNA at target site in CRISPR-mediated gene editing( shown in Figure 2E). In some cases wild-type Cas9 can cleave other genomic sites with several nucleotides difference to on-target site, inducing unwanted off-target mutations(Cho et al., 2014; Cong et al., 2013; Fu et al., 2013).

Secondly, mutated Cas9 (Cas9n) with one point mutation either H840A in HNH domain or D10A in the RuvC domain, which were found to be more specific and efficient for genome targeting purposes(Ran et al., 2013)( shown in Figure 2F).Cas9n has the ability to create a nick instead of a DSB at the target site (Cong et al., 2013; Ran et al., 2013). The nicks are mostly repaired by the high-fidelity base excision pathway and can therefore increase the specificity of CRISPR/Cas system (Cong et al., 2013). Additionally, a pair of double nicks at two sites by four customized gRNAs/Cas complex successfully deleted genomic fragments of up to $6 \mathrm{~kb}$ in HEK 293FT cells (Ran et al., 2013). Cas9n reduces the off-targeting by 50 - to 1,000 -fold without sacrificing the on-target effect (Ran et al., 2013). Thus combination of two gRNAs is preferred when the specificity is of paramount importance (e.g. the creation of engineered cell lines). Meanwhile the delivery of two gRNAs reduces the likelihood that their off-target sites are close enough to cause double stranded breaks.

The last one is dead Cas9 (dCas9) which is generated by double point mutations of $\mathrm{H} 840 \mathrm{~A}$ in the $\mathrm{HNH}$ domain and D10A in the RuvC domain (Figure $2 \mathrm{G}$ ). The dCas9 protein lost the cutting ability yet retained the DNA binding ability (Gasiunas et al., 2012; Jinek et al., 2012). Therefore, this catalytically dead Cas 9 variant can be used for the control of gene expression. Repression of gene expression by dCas9 based CRISPR interference (CRISPRi) is proven to be more significant than classic RNAi-based silencing. CRISPRi regulate the gene expression at transcriptional level by blocking RNA polymerase, transcription initiation and elongation, whereas RNA interference controls gene function at mRNA level. The effect of repression can be further enhanced by fusing dCas 9 with transcription effectors. KRAB, a transcriptional repressive domain fused with dCas9, showed a high repression efficiency compared to dCas9 in HEK293 cells (Gilbert et al., 2013). CRISPR/ Cas system has also been used to activate and up-regulate genes expression, which is termed as CRISPRa(Maeder et al., 2013). It is reported that the fusion of dCas9 with VP64, 
Table 3. Web-based tools for sgRNA design.

\begin{tabular}{|c|c|c|}
\hline Name & Developer & Web address \\
\hline SgRNA designer & Broad Institute & $\begin{array}{l}\text { http://www.broadinstitute.org/rnai/public/analysis- } \\
\text { tools/sgrna-design }\end{array}$ \\
\hline Zinc finger Consortium & Keith Joung lab, Harvard University & http://zifit.partners.org/ZiFiT/ChoiceMenu.aspx \\
\hline ChopChop & $\begin{array}{l}\text { George church lab, Harvard } \\
\text { University }\end{array}$ & https://chopchop.rc.fas.harvard.edu/ \\
\hline ССТор & $\begin{array}{l}\text { Center for organismal studies, } \\
\text { Heidelberg university. }\end{array}$ & http://crispr.cos.uni-heidelberg.de/ \\
\hline CRISPR Design & $\begin{array}{l}\text { Feng Zhang lab, Massachusetts } \\
\text { Institute of Technology }\end{array}$ & http://crispr.mit.edu/ \\
\hline E-CRISPR & German cancer research center & http://www.e-crisp.org/E-CRISP/ \\
\hline CasOT & $\begin{array}{l}\text { Zebrafish functional genomics } \\
\text { group Peking university }\end{array}$ & http://eendb.zfgenetics.org/c=asot/ \\
\hline
\end{tabular}

VP65 or omega can increased the rate of gene expression by 25-fold (Gilbert et al., 2013). The level of activation depends upon the distance between dCas9 binding site and promoter (Bikard et al., 2013).

Several researches have shown that the fusion of Fokl nuclease domain to dCas9 could improve targeting specificity and largely reduce the off-target effect (Guilinger et al., 2014; Wyvekens et al., 2015). The complex of two Fokl-dCas9 fusions and two gRNAs, known as RFNs (RNA-guided Fokl nucleases), is very specific in gene editing as the RFNs complex does not induce any DNA lesions.dCas9 itself binds to the target site, while the fused Fok1 endonuclease cut the double stranded DNA dictated by a pair of gRNAs ( as shown in Figure 2H). RFN cleavage activity strictly depends on the binding of two gRNAs onto target DNA. The DNA cleavage requires the PAM sites to be a certain distance apart and in a particular orientation with respect to each other (Tsai et al., 2014). RFNs offers a better recognition fidelity and greater specificity than Cas9 and Cas9n (Guilinger et al., 2014). However, the problem of narrow target site selection is significant, which may be tolerable for making Knock-outs but challenging for specific knock-in.

\section{DNA repair process in CRISPR-mediated gene editing}

Following the cleavage by Cas9, the repair process of double stranded breaks is critical for CRISPR efficiency, which is necessary to be concerned. The repairing pathways include homology directed repair (HDR) and nonhomologous end joining (NHEJ).NHEJ has been shown to be dominant during $\mathrm{G} 1, \mathrm{~S}$ and $\mathrm{G} 2$ phases while homology directed repair (HDR) dominates in late $S$ and G2 phase (Heyer et al., 2010). These two pathways have been manipulated by researchers for genome editing using CRISPR in mammalian cell (Cong et al., 2013; Mali et al., 2013b) (shown in Figure 1).
To date, CRISPR-mediated gene knockout through NHEJ induced insertion-deletion (Indels) mutations has been employed efficiently, with a frequency of $20 \%-60 \%$ in mouse embryonic stem cells (ESCs) and zygotes (Wang et al., 2013; Yang et al., 2013). However insertion of point mutation or gene at precise position by HDR remains inefficient (Mali et al., 2013b; Wang et al., 2013; Yang et al., 2013).Indel mutations induced by NHEJ at the cleaved site may lead to frame shift mutation and deletion, ultimately resulting in the malfunction of the gene (Bibikova et al., 2002). As HDR mediated gene editing is more precise, the specificity of genome editing could be increased by maximizing the chances of repairing by HDR. In this line, Cas9n with single gRNA activates HDR and thus reduces off-target potentials (Cong et al., 2013). However, NHEJ will compete with HDR to rectify double stranded breaks. In order to promote HDR, researchers have used inhibitors of the NHEJ repair pathway and achieved higher efficiency of gene editing. When DNA ligase IV (a principal enzyme involved in NHEJ repair pathway) was antagonized by its inhibitor Scr7, the genome editing efficiency was increased up to 19 fold(Maruyama et al., 2015). Under the gene silencing of NHEJ key molecules or co-expression of adenovirus E1B55K and E4orf6 protein, the efficiency of HDR was increased accompanying abolished NHEJ activity in both human and mouse cell lines (Chu et al., 2015). The efficiency of HDR is highly dependent on the cell type, state and genomic content (Saleh-Gohari and Helleday, 2004). Cell cycle synchronization in late G2 phase increases HDR efficiency and reduces unwanted NHEJ events (Lin et al., 2015). The specificity and accuracy of the HDR-based gene editing process also depends upon the nature of donor DNA(Holkers et al., 2014). If the foreign DNA have a homology more than 400bp with the target, it will lead to more efficient introduction of precise nucleotide substitutions or deletions, (McMahon et al., 2012). 


\section{Selection of unique target site in the genome}

Sequence features control the specificity of CRISPR/Cas mediated gene editing. Genomic sequences with similarity to target site would be more vulnerable to mutation. To create a loss-of-function mutation, designing the gRNA to target $\mathrm{N}$-terminus of the gene is more successful. For gene activation, it is important to design gRNA in the proximity of the transcription start site at a favorable location in the promoter region to facilitate the easy access of transcriptional factors. For gene repression, gRNA targeting the coding region is more efficient $(\geq 80 \%)$, while there is no repression effect when it binds to antisense strand (Bikard et al., 2013; Choudhary et al., 2015).

\section{Optimization of method for Cas-sgRNA delivery and expression}

The delivery and transient expression of CRISPR/Cas system are needed to be optimized for each particular cell type or organism. Appropriate methods developed to efficiently deliver Cas9 and gRNA to target cell or tissue could potentially increase CRISPR specificity. Expression of the Cas9 and gRNA complex in cultured mammalian cells is mostly achieved by electroporation, microinjection, lipofection and cell penetetrating peptides. Delivery of CassgRNA via plasmids transfection could cause overexpression of Cas9 which will increase off-target mutation rate. Viral vectors such as Adeno-associated virus (AAV) and lentivirus are broadly used as gene delivering tools. $\mathrm{AAV}$ and lentivirus based gene delivery is beneficial for their high efficacy and low pathogenicity. However the large size of SpCas9 (4.2 kb in length) will cause low titer production of AAV. This was resolved by a smaller size SaCas9 (3.3kb) (Ran et al., 2015).

The delivery of Cas/gRNA to cells by microinjection has induced site specific mutation up to $79 \%$ and reduced offtarget cleavage (Kim et al., 2014; Lee et al., 2014). This method has some advantages as this complex provides a controlled Cas9 amount to the cell followed by rapid degradation. The invention of safe and improved delivery method of Cas9-sgRNA is urgently needed to enable more efficient and specific genome editing.

As discussed above, the method for delivery of the CRISPR system is of great concern as it allows controlled optimum concentration of Cas9 and sgRNA in a cell that in turns important for increasing the specificity (Hsu et al., 2013; Pattanayak et al., 2013). Recently, a positive correlation was observed between Cas9/sgRNA expression level and mutagenesis efficiency in plants (Mikami et al., 2015), as indicated by increased specificity when lowering the amount of plasmid DNA in transfection. The effect of alteration in the enzyme concentration is varied at different PAM positions. Under normal enzymatic concentrations, the proximal region to PAM is regarded as highly specific (Cong et al., 2013; Jinek et al., 2012; Sapranauskas et al., 2011). Reduction in the enzyme concentration achieve most specificity $(\leq 80 \%)$ at the PAM proximal end compared to the PAM distal end (30\%) (Pattanayak et al., 2013). While in excessive enzyme concentration, mismatches in these regions are tolerated (Pattanayak et al., 2013). However, it is noteworthy that reducing the Cas9/gRNA complex also decreases the on-target site cleavage efficiency (Pattanayak et al., 2013; Ran et al., 2013). The use of a weak or inducible promoter is thought to regulate the cas9 expression to optimal level. The constitutive expression of these endonuclease increase the ontargeting editing but might also leads to higher off-target effect, as observed for zinc finger nucleases (ZFNs) (Gaj et al., 2012). The expression timing of Cas9 is an additional factor affecting off-target, because short lived and timed delivery of Cas9 allowed minimum off-target effects (Lin et al., 2015).

\section{Analyzing off-target effect}

Prediction of off-target sites is difficult, as the off-target cleavage is not fully understood. Meanwhile, the methods for off-target analysis have low sensitivity and lack unbiased genome wide characterization. Various methods including restriction fragment length polymorphism (RFLP), PCR amplification of off-target sites followed by next generation sequencing (NGS), or mismatch sensitive nuclease (Surveyor assay and T7 endonuclease assay) have been used to detect the off-target mutations. The T7 endonuclease assay is more sensitive with deletion substrates, while surveyor is suited for detecting single nucleotide changes. For endonuclease assays, it is challenging to detect Indels below $1 \%$. It is also possible to sequence the PCR product using next generation sequencing (NGS), which is more sensitive and accurate but very expensive.

The methods used to detect off-target sites are mostly based on characterizations of the nucleases used to binding site or DNA cutting assay in vitro or in vivo. The methods assaying Cas9 binding include in cellulo ChIP (chromatin immunoprecipitation) and invitro SELEX (systematic evolution of ligands by exponential amplification) (shown in Figure 3). Double stranded breaks(DSBs) capturing methods including IDLV (Integrase deficient Lenti virus) and BLESS (breaks labeling enrichment on streptavidin and next generation sequencing) have also been applied to detect off-target sites (shown in Figure 3). IDLV can incorporate into the DSBs via non-homologous end joining (NHEJ) and tag those transient DSBs(Wang et al., 2015) Another IDLV method is based on in vitro cleavage of a library followed by deep sequencing. BLESS detects in vivo DNA cleavage by in vitro DSB capturing method which involves fixation of cells, chromatin purification, nuclease digestion, ligation with biotenylated linkers and targeted deep sequencing (Ran et al., 2015). The BLESS method has poor sensitivity as many bona fide off-target sites are missed. Meanwhile detecting mutation at single sites and single instant by BLESS method is also susceptible to artifacts associated with cell fixation (Tsai et al., 2015). The ChIP assay is poorly sensitive to detect low level of off-target sites and shows high rate of false positive detection (Kuscu et al., 2014; O'Geen et al., 2014; Wu et al., 2014). The ChIPseq assay has advantage in revealing genome wide binding of dCas9, but it cannot predict off-target binding and cleaving sites of catalytically active Cas9. 


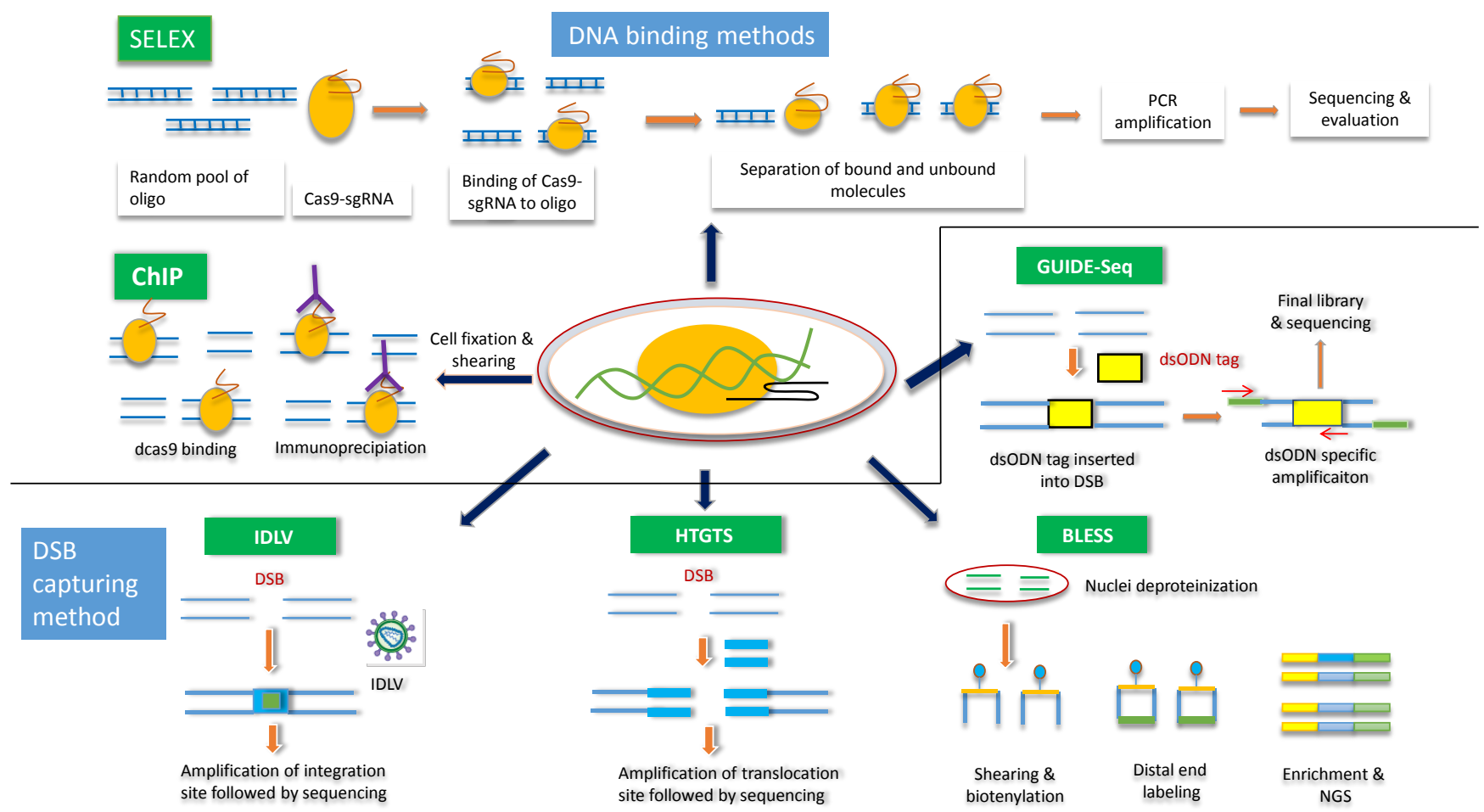

Figure 3. Schematic overview of method used for assaying off-target sites induced by CRISPR system. Center: genomic DNA in the cell cleaved by Cas9. DSB capturing methods including IDLV and BLESS. DNA binding methods including ChIP-seq and SELEX.

Alternatively, method like Genome-wide Unbiased Indentification of DSBs evaluated by Sequencing (GUIDEseq)is recently developed. GUIDE-seq and high-throughput genome-wide translocation sequencing (HTGTS) have proved to be more reliable and accurate tools (shown in Figure 3). GUIDE-seq works by incorporation of 34 bp double-stranded phopho thiorate oligo deoxy nucleotides (dsODN) into the DSB via NHEJ, selective amplification of targets site with some portion of dsODN and construction of library containing high throughput sequences. The process indicated the number of off-target sites with a frequency of $0.1 \%$ (Tsai et al., 2015). HTGTS relies on creating a DNA break in a known site of the genome and using it as bait to capture prey DNA that have naturally broken off in other parts of the genome. High throughput sequencing is used to identify prey sequences. More recently, another unbiased in vitro digestion method, digested genome sequencing (DGS), has been developed to exploit in vitro digestion by Cas 9 followed by whole genome sequencing. The digested sites have same 5 'end determined by deep sequencing. The methods (HGST, GUIDE-seq and Digenome-seq) exploit the use of single gRNA targeting a single locus VEGF-A. HTGTS and GUIDE-seq detected a set of 7 off-target sites, conversely Digenome-seq showed 8 off-target site with an indel frequency below $1 \%(0.065 \%)$ detected by deep sequencing (Koo et al., 2015).Finally, as one cannot predict which method is the best way to locate off-target sites, it is deal to run multiple methods in parallel for the same nuclease. For example, coupling ChIP in vitro selection with whole genome sequencing will help to avoid false positive. Similarly, linking DSBs capture method with bar code linkers allows multiple gRNA study at a time.

\section{Summary and perspectives}

The CRISPR/Cas, a natural bacterial immune system, is reconstructed as a robust platform for engineering biology with simplicity and efficiency CRISPR is widely and successfully applied in genome editing but it is still a developing system, requiring target specificity optimization. In this regard, the development of smart strategies to overcome the non-specific editing problems is desperately needed. The integration of these strategies discussed in this review to improve CRISPR/Cas specificity focus on judicious selection of target sites and gRNAs from a diverse range of target sites, Cas 9 protein engineering, discovering new Cas9 orthologs with efficient low-cost assembly as well as the optimization of effective safe delivery systems. Moreover, understanding the basic recombination and repair machinery of DNA would contribute to improve CRISPR specificity. In future, the progress of modern molecular biology will eventually equip CRISPR/Cas system with high specificity and no off-target effects and pave the way for therapeutic strategies without side effects.

\section{Acknowledgments}

This work was supported by the National Natural Science F oundation of China (Grant No.31371106, 31421064), Hubei Provincial Natural Science Foundation of China (Grant No. 36113011), Doctoral Fund of Ministry of Education of China (Grant No. 131012) and the 
Huazhong Agricultural University Scientific \&Technological Self-innovation Foundation (Program No. 52204-13002). The authors are highly thankful to Dr. Denis Headon and Dr. Jonathan D Moore for their assistance.

\section{References}

Auer, T.O., Duroure, K., De Cian, A., Concordet, J.-P., and Del Bene, F. (2014). Highly efficient CRISPR/Cas9mediated knock-in in zebrafish by homology-independent DNA repair. Genome research 24, 142-153.

Belhaj, K., Chaparro-Garcia, A., Kamoun, S., and Nekrasov, V. (2013). Plant genome editing made easy: targeted mutagenesis in model and crop plants using the CRISPR/Cas system. Plant methods 9, 39.

Bhaya, D., Davison, M., and Barrangou, R. (2011). CRISPR-Cas systems in bacteria and archaea: versatile small RNAs for adaptive defense and regulation. Annual review of genetics 45, 273-297.

Bibikova, M., Golic, M., Golic, K.G., and Carroll, D. (2002). Targeted chromosomal cleavage and mutagenesis in Drosophila using zinc-finger nucleases. Genetics 161, 1169-1175.

Bikard, D., Jiang, W., Samai, P., Hochschild, A., Zhang, F., and Marraffini, L.A. (2013). Programmable repression and activation of bacterial gene expression using an engineered CRISPR-Cas system. Nucleic Acids Research 41, 7429-7437.

Blitz, I.L., Biesinger, J., Xie, X., and Cho, K.W. (2013). Biallelic genome modification in F0 Xenopus tropicalis embryos using the CRISPR/Cas system. Genesis 51, 827-834.

Carroll, D. (2014). Genome engineering with targetable nucleases. Annual review of biochemistry 83, 409-439.

Chen, B., Gilbert, L.A., Cimini, B.A., Schnitzbauer, J., Zhang, W., Li, G.-W., Park, J., Blackburn, E.H., Weissman, J.S., and Qi, L.S. (2013). Dynamic imaging of genomic loci in living human cells by an optimized CRISPR/Cas system. Cell 155, 1479-1491.

Cho, S.W., Kim, S., Kim, Y., Kweon, J., Kim, H.S., Bae, S., and Kim, J.-S. (2014). Analysis of off-target effects of CRISPR/Cas-derived RNA-guided endonucleases and nickases. Genome Research 24, 132-141.

Choudhary, E., Thakur, P., Pareek, M., and Agarwal, N. (2015). Gene silencing by CRISPR interference in mycobacteria. Nature communications 6 .

Chu, V.T., Weber, T., Wefers, B., Wurst, W., Sander, S., Rajewsky, K., and Kuhn, R. (2015). Increasing the efficiency of homology-directed repair for CRISPR-Cas9induced precise gene editing in mammalian cells. Nat Biotech 33, 543-548.

Cong, L., Ran, F.A., Cox, D., Lin, S., Barretto, R., Habib, N., Hsu, P.D., Wu, X., Jiang, W., and Marraffini, L.A. (2013). Multiplex genome engineering using CRISPR/ Cas systems. Science 339, 819-823.

Cradick, T.J., Fine, E.J., Antico, C.J., and Bao, G. (2013). CRISPR/Cas9 systems targeting $\beta$-globin and CCR5 genes have substantial off-target activity. Nucleic acids research, gkt714.

Daimon, T., Kiuchi, T., and Takasu, Y. (2014). Recent progress in genome engineering techniques in the silkworm, Bombyx mori. Development, growth \& differentiation 56, 14-25.
Deveau, H., Barrangou, R., Garneau, J.E., Labonté, J., Fremaux, C., Boyaval, P., Romero, D.A., Horvath, P., and Moineau, S. (2008). Phage response to CRISPRencoded resistance in Streptococcus thermophilus. Journal of bacteriology 190, 1390-1400.

Deveau, H., Garneau, J.E., and Moineau, S. (2010). CRISPR/Cas system and its role in phage- bacteria interactions. Annual review of microbiology 64, 475-493.

Doench, J.G., Hartenian, E., Graham, D.B., Tothova, Z., Hegde, M., Smith, I., Sullender, M., Ebert, B.L., Xavier, R.J., and Root, D.E. (2014). Rational design of highly active sgRNAs for CRISPR-Cas9-mediated gene inactivation. Nat Biotech 32, 1262-1267.

Flowers, G.P., Timberlake, A.T., Mclean, K.C., Monaghan, J.R., and Crews, C.M. (2014). Highly efficient targeted mutagenesis in axolotl using Cas9 RNA-guided nuclease. Development 141, 2165-2171.

Fu, Y., Foden, J.A., Khayter, C., Maeder, M.L., Reyon, D., Joung, J.K., and Sander, J.D. (2013). High-frequency offtarget mutagenesis induced by CRISPR-Cas nucleases in human cells. Nature biotechnology 31, 822-826.

Fu, Y., Sander, J.D., Reyon, D., Cascio, V.M., and Joung, J.K. (2014). Improving CRISPR-Cas nuclease specificity using truncated guide RNAs. Nature biotechnology 32, 279-284.

Fujii, W., Kawasaki, K., Sugiura, K., and Naito, K. (2013). Efficient generation of large-scale genome-modified mice using gRNA and CAS9 endonuclease. Nucleic Acids Research 41, e187.

Gaj, T., Guo, J., Kato, Y., Sirk, S.J., and Barbas, C.F. (2012). Targeted gene knockout by direct delivery of zincfinger nuclease proteins. Nat Meth 9, 805-807.

Gasiunas, G., Barrangou, R., Horvath, P., and Siksnys, V. (2012). Cas9-crRNA ribonucleoprotein complex mediates specific DNA cleavage for adaptive immunity in bacteria. Proceedings of the National Academy of Sciences of the United States of America 109, E2579-E2586.

Gilbert, L.A., Larson, M.H., Morsut, L., Liu, Z., Brar, G.A., Torres, S.E., Stern-Ginossar, N., Brandman, O., Whitehead, E.H., and Doudna, J.A. (2013). CRISPRmediated modular RNA-guided regulation of transcription in eukaryotes. Cell 154, 442-451.

Gratz, S.J., Wildonger, J., Harrison, M.M., and O'ConnorGiles, K.M. (2013). CRISPR/Cas9-mediated genome engineering and the promise of designer flies on demand. Fly 7, 249-255.

Gudbergsdottir, S., Deng, L., Chen, Z., Jensen, J.V., Jensen, L.R., She, Q., and Garrett, R.A. (2011). Dynamic properties of the Sulfolobus CRISPR/Cas and CRISPR/ $\mathrm{Cmr}$ systems when challenged with vector-borne viral and plasmid genes and protospacers. Molecular microbiology 79, 35-49.

Guilinger, J.P., Thompson, D.B., and Liu, D.R. (2014). Fusion of catalytically inactive Cas 9 to Fokl nuclease improves the specificity of genome modification. Nat Biotech 32, 577-582.

Guo, X., Zhang, T., Hu, Z., Zhang, Y., Shi, Z., Wang, Q., Cui, Y., Wang, F., Zhao, H., and Chen, Y. (2014). Efficient RNA/Cas9-mediated genome editing in Xenopus tropicalis. Development 141, 707-714. 
Guilinger, J.P., Thompson, D.B., and Liu, D.R. (2014). Fusion of catalytically inactive Cas9 to Fokl nuclease improves the specificity of genome modification. Nat Biotech 32, 577-582.

Guo, X., Zhang, T., Hu, Z., Zhang, Y., Shi, Z., Wang, Q., Cui, Y., Wang, F., Zhao, H., and Chen, Y. (2014). Efficient RNA/Cas9-mediated genome editing in Xenopus tropicalis. Development 141, 707-714.

Hai, T., Teng, F., Guo, R., Li, W., and Zhou, Q. (2014). Onestep generation of knockout pigs by zygote injection of CRISPR/Cas system. Cell research 24, 372.

Heigwer, F., Kerr, G., and Boutros, M. (2014). E-CRISP: fast CRISPR target site identification. Nat Meth 11, 122-123.

Holkers, M., Maggio, I., Henriques, S.F.D., Janssen, J.M., Cathomen, T., and Goncalves, M.A.F.V. (2014). Adenoviral vector DNA for accurate genome editing with engineered nucleases. Nat Meth 11, 1051-1057.

Horvath, P., and Barrangou, R. (2010). CRISPR/Cas, the immune system of bacteria and archaea. Science 327 , 167-170.

Hou, Z., Zhang, Y., Propson, N.E., Howden, S.E., Chu, L.F., Sontheimer, E.J., and Thomson, J.A. (2013). Efficient genome engineering in human pluripotent stem cells using Cas9 from Neisseria meningitidis. Proceedings of the National Academy of Sciences 110, 15644-15649

Hsu, P.D., Scott, D.A., Weinstein, J.A., Ran, F.A., Konermann, S., Agarwala, V., Li, Y., Fine, E.J., Wu, X., and Shalem, O. (2013). DNA targeting specificity of RNAguided Cas 9 nucleases. Nature biotechnology 31, 827-832.

Hwang, W.Y., Fu, Y., Reyon, D., Maeder, M.L., Kaini, P., Sander, J.D., Joung, J.K., Peterson, R.T., and Yeh, J.R.J. (2013). Heritable and precise zebrafish genome editing using a CRISPR-Cas system. PloS one 8, e68708.

Ishino, Y., Shinagawa, H., Makino, K., Amemura, M., and Nakata, A. (1987). Nucleotide sequence of the iap gene, responsible for alkaline phosphatase isozyme conversion in Escherichia coli, and identification of the gene product. Journal of bacteriology 169, 5429-5433.

Jia, H., and Wang, N. (2014). Targeted genome editing of sweet orange using Cas9/sgRNA. PLoS One 9, e93806.

Jiang, W., Bikard, D., Cox, D., Zhang, F., and Marraffini, L.A. (2013a). CRISPR-assisted editing of bacterial genomes. Nature biotechnology 31, 233.

Jiang, W., Bikard, D., Cox, D., Zhang, F., and Marraffini, L.A. (2013b). RNA-guided editing of bacterial genomes using CRISPR-Cas systems. Nat Biotech 31, 233-239.

Jiang, W., Zhou, H., Bi, H., Fromm, M., Yang, B., and Weeks, D.P. (2013c). Demonstration of CRISPR/Cas9/ sgRNA-mediated targeted gene modification in Arabidopsis, tobacco, sorghum and rice. Nucleic acids research, gkt780.

Jinek, M., Chylinski, K., Fonfara, I., Hauer, M., Doudna, J.A., and Charpentier, E. (2012). A Programmable DualRNA-Guided DNA Endonuclease in Adaptive Bacterial Immunity. Science 337, 816-821.

Kim, D., Bae, S., Park, J., Kim, E., Kim, S., Yu, H.R., Hwang, J., Kim, J.-I., and Kim, J.-S. (2015). Digenomeseq: genome-wide profiling of CRISPR-Cas9 off-target effects in human cells. Nat Meth 12, 237-243.
Kim, S., Kim, D., Cho, S.W., Kim, J., and Kim, J.-S. (2014). Highly efficient RNA-guided genome editing in human cells via delivery of purified Cas9 ribonucleoproteins. Genome research 24, 1012-1019.

Koo, T., Lee, J., and Kim, J. (2015). Measuring and Reducing Off-Target Activities of Programmable Nucleases Including CRISPR-Cas9. Molecules and cells.

Koonin, E.V., and Makarova, K.S. (2009). CRISPR-Cas: an adaptive immunity system in prokaryotes. F1000 biology reports 1.

Kuscu, C., Arslan, S., Singh, R., Thorpe, J., and Adli, M. (2014). Genome-wide analysis reveals characteristics of off-target sites bound by the Cas 9 endonuclease. Nat Biotech 32, 677-683.

Lee, J.-S., Kwak, S.-J., Kim, J., Kim, A.-K., Noh, H.M., Kim, J.-S., and Yu, K. (2014). RNA-guided genome editing in Drosophila with the purified Cas9 protein. G3: Genes| Genomes| Genetics 4, 1291-1295.

Li, W., Teng, F., Li, T., and Zhou, Q. (2013). Simultaneous generation and germline transmission of multiple gene mutations in rat using CRISPR-Cas systems. Nat Biotech 31, 684-686.

Liang, Z., Zhang, K., Chen, K., and Gao, C. (2014). Targeted mutagenesis in Zea mays using TALENs and the CRISPR/Cas system. Journal of Genetics and Genomics 41, 63-68.

Lillestøl, R.K., Shah, S.A., Brügger, K., Redder, P., Phan, H., Christiansen, J., and Garrett, R.A. (2009). CRISPR families of the crenarchaeal genus Sulfolobus: bidirectional transcription and dynamic properties. Molecular microbiology 72, 259-272.

Lin, S., Staahl, B.T., Alla, R.K., and Doudna, J.A. (2015). Enhanced homology-directed human genome engineering by controlled timing of CRISPR/Cas9 delivery. Elife 3, e04766.

Maeder, M.L., Linder, S.J., Cascio, V.M., Fu, Y., Ho, Q.H., and Joung, J.K. (2013). CRISPR RNA-guided activation of endogenous human genes. Nature methods 10 , 977-979.

Mali, P., Aach, J., Stranges, P.B., Esvelt, K.M., Moosburner, M., Kosuri, S., Yang, L., and Church, G.M. (2013a). CAS9 transcriptional activators for target specificity screening and paired nickases for cooperative genome engineering. Nature biotechnology 31, 833-838.

Mali, P., Yang, L., Esvelt, K.M., Aach, J., Guell, M., DiCarlo, J.E., Norville, J.E., and Church, G.M. (2013b). RNAguided human genome engineering via Cas9. Science 339, 823-826.

Manica, A., Zebec, Z., Teichmann, D., and Schleper, C. (2011). In vivo activity of CRISPR-mediated virus defence in a hyperthermophilic archaeon. Molecular microbiology 80, 481-491.

Maruyama, T., Dougan, S.K., Truttmann, M.C., Bilate, A.M., Ingram, J.R., and Ploegh, H.L. (2015). Increasing the efficiency of precise genome editing with CRISPR-Cas9 by inhibition of nonhomologous end joining. Nat Biotech 33, 538-542.

McMahon, M.A., Rahdar, M., and Porteus, M. (2012). Gene editing: not just for translation anymore. Nature methods $9,28-31$. 
Mikami, M., Toki, S., and Endo, M. (2015). Parameters affecting frequency of CRISPR/Cas 9 mediated targeted mutagenesis in rice. Plant cell reports, 1-9.

Nishimasu, H., Ran, F.A., Hsu, P.D., Konermann, S., Shehata, S.I., Dohmae, N., Ishitani, R., Zhang, F., and Nureki, O. (2014). Crystal structure of Cas9 in complex with guide RNA and target DNA. Cell 156, 935-949.

Niu, Y., Shen, B., Cui, Y., Chen, Y., Wang, J., Wang, L., Kang, Y., Zhao, X., Si, W., and Li, W. (2014). Generation of gene-modified cynomolgus monkey via Cas9/RNAmediated gene targeting in one-cell embryos. Cell 156, 836-843.

O'Geen, H., Henry, I.M., Bhakta, M.S., Meckler, J.F., and Segal, D.J. (2014). A genome-wide analysis of Cas9 binding specificity using ChIP-seq and targeted sequence capture.

Oh, J.-H., and van Pijkeren, J.-P. (2014). CRISPR-Cas9assisted recombineering in Lactobacillus reuteri. Nucleic acids research, gku623

Pattanayak, V., Lin, S., Guilinger, J.P., Ma, E., Doudna, J.A., and Liu, D.R. (2013). High-throughput profiling of off-target DNA cleavage reveals RNA-programmed Cas9 nuclease specificity. Nature biotechnology 31, 839-843.

Perez-Pinera, P., Kocak, D.D., Vockley, C.M., Adler, A.F., Kabadi, A.M., Polstein, L.R., Thakore, P.I., Glass, K.A., Ousterout, D.G., and Leong, K.W. (2013). RNA-guided gene activation by CRISPR-Cas9-based transcription factors. Nature methods 10, 973-976.

Ran, F.A., Cong, L., Yan, W.X., Scott, D.A., Gootenberg, J.S., Kriz, A.J., Zetsche, B., Shalem, O., Wu, X., and Makarova, K.S. (2015). In vivo genome editing using Staphylococcus aureus Cas9. Nature 520, 186-191.

Ran, F.A., Hsu, P.D., Lin, C.-Y., Gootenberg, J.S., Konermann, S., Trevino, A.E., Scott, D.A., Inoue, A., Matoba, S., and Zhang, Y. (2013). Double nicking by RNA-guided CRISPR Cas9 for enhanced genome editing specificity. Cell 154, 1380-1389.

Saleh-Gohari, N., and Helleday, T. (2004). Conservative homologous recombination preferentially repairs DNA double-strand breaks in the $S$ phase of the cell cycle in human cells. Nucleic acids research 32, 3683-3688.

Sander, J.D., and Joung, J.K. (2014). CRISPR-Cas systems for editing, regulating and targeting genomes. Nature biotechnology 32, 347-355.

Sander, J.D., Maeder, M.L., Reyon, D., Voytas, D.F., Joung, J.K., and Dobbs, D. (2010). ZiFiT (Zinc Finger Targeter): an updated zinc finger engineering tool. Nucleic Acids Research 38, W462-W468.

Sapranauskas, R., Gasiunas, G., Fremaux, C., Barrangou, R., Horvath, P., and Siksnys, V. (2011). The Streptococcus thermophilus CRISPR/Cas system provides immunity in Escherichia coli. Nucleic acids research, gkr606.

Shan, Q., Wang, Y., Li, J., Zhang, Y., Chen, K., Liang, Z., Zhang, K., Liu, J., Xi, J.J., Qiu, J.-L., et al. (2013). Targeted genome modification of crop plants using a CRISPR-Cas system. Nat Biotech 31, 686-688.

Sugano, S.S., Shirakawa, M., Takagi, J., Matsuda, Y., Shimada, T., Hara-Nishimura, I., and Kohchi, T. (2014). CRISPR/Cas9 Mediated Targeted Mutagenesis in the Liverwort Marchantia polymorpha L. Plant and Cell Physiology, pcu014.
Sugimoto, N., Nakano, S.-i., Katoh, M., Matsumura, A., Nakamuta, H., Ohmichi, T., Yoneyama, M., and Sasaki, M. (1995). Thermodynamic parameters to predict stability of RNA/DNA hybrid duplexes. Biochemistry 34, 11211-11216.

Terns, M.P., and Terns, R.M. (2011). CRISPR-based adaptive immune systems. Current opinion in microbiology 14, 321-327.

Tsai, S.Q., Wyvekens, N., Khayter, C., Foden, J.A., Thapar, V., Reyon, D., Goodwin, M.J., Aryee, M.J., and Joung, J.K. (2014). Dimeric CRISPR RNA-guided FokI nucleases for highly specific genome editing. Nature biotechnology 32, 569-576.

Tsai, S.Q., Zheng, Z., Nguyen, N.T., Liebers, M., Topkar, V.V., Thapar, V., Wyvekens, N., Khayter, C., lafrate, A.J., and Le, L.P. (2015). GUIDE-seq enables genome-wide profiling of off-target cleavage by CRISPR-Cas nucleases. Nature biotechnology 33, 187-197.

Upadhyay, S.K., Kumar, J., Alok, A., and Tuli, R. (2013). RNA-guided genome editing for target gene mutations in wheat. G3: Genes| Genomes| Genetics 3, 2233-2238.

van der Ploeg, J.R. (2009). Analysis of CRISPR in Streptococcus mutans suggests frequent occurrence of acquired immunity against infection by M102-like bacteriophages. Microbiology 155, 1966-1976.

Waaijers, S., and Boxem, M. (2014). Engineering the Caenorhabditis elegans genome with CRISPR/Cas9. Methods 68, 381-388.

Wang, H., Yang, H., Shivalila, Chikdu S., Dawlaty, Meelad M., Cheng, Albert W., Zhang, F., and Jaenisch, R. (2013). One-Step Generation of Mice Carrying Mutations in Multiple Genes by CRISPR/Cas-Mediated Genome Engineering. Cell 153, 910-918.

Wang, T., Wei, J.J., Sabatini, D.M., and Lander, E.S. (2014). Genetic Screens in Human Cells Using the CRISPR-Cas9 System. Science 343, 80-84.

Wang, X., Wang, Y., Wu, X., Wang, J., Wang, Y., Quu, Z., Chang, T., Huang, H., Lin, R.-J., and Yee, J.-K. (2015). Unbiased detection of off-target cleavage by CRISPRCas9 and TALENs using integrase-defective lentiviral vectors. Nat Biotech 33, 175-178.

Wang, Y., Li, Z., Xu, J., Zeng, B., Ling, L., You, L., Chen, Y., Huang, Y., and Tan, A. (2013b). The CRISPR/Cas system mediates efficient genome engineering in Bombyx mori. Cell research 23, 1414 .

Wiedenheft, B., Sternberg, S.H., and Doudna, J.A. (2012). RNA-guided genetic silencing systems in bacteria and archaea. Nature 482, 331-338.

Wu, X., Scott, D.A., Kriz, A.J., Chiu, A.C., Hsu, P.D., Dadon, D.B., Cheng, A.W., Trevino, A.E., Konermann, S., Chen, S., et al. (2014). Genome-wide binding of the CRISPR endonuclease Cas9 in mammalian cells. Nat Biotech 32, 670-676.

Wyvekens, N., Topkar, V.V., Khayter, C., Joung, J.K., and Tsai, S.Q. (2015). Dimeric CRISPR RNA-guided FokldCas9 nucleases (RFNs) directed by truncated gRNAs for highly specific genome editing. Human gene therapy.

Yang, D., Xu, J., Zhu, T., Fan, J., Lai, L., Zhang, J., and Chen, Y.E. (2014). Effective gene targeting in rabbits using RNA-guided Cas9 nucleases. Journal of molecular cell biology $6,97-99$. 
Yang, H., Wang, H., Shivalila, Chikdu S., Cheng, Albert W., Shi, L., and Jaenisch, R. (2013). One-Step Generation of Mice Carrying Reporter and Conditional Alleles by CRISPR/Cas-Mediated Genome Engineering. Cell 154, 1370-1379. 


\section{Microbiology / Molecular Biology}

Caister Academic Press is a leading academic publisher of advanced texts in microbiology, molecular biology and medical research. Full details of all our publications at caister.com

- Cyanobacteria: Omics and Manipulation Edited by: DA Los (2017) www.caister.com/cyano3

- Brain-eating Amoebae: Biology and Pathogenesis of Naegleria fowleri

Author: R Siddiqui, IKM Ali, JR Cope, et al. (2016)

"explains the current knowledge and research" (ProtoView) www.caister.com/naegleria

- Foot and Mouth Disease Virus: Current Research and Emerging Trends

Edited by: F Sobrino, E Domingo (2017)

www.caister.com/fmdv

- Staphylococcus: Genetics and Physiology

Edited by: GA Somerville (2016)

www.caister.com/staph2

- Chloroplasts: Current Research and Future Trends Edited by: H Kirchhoff (2016)

www.caister.com/chloroplasts

- Microbial Biodegradation: From Omics to Function and Application

Edited by: J Długoński (2016)

www.caister.com/biodegradation

- Influenza: Current Research

Edited by: Q Wang, YJ Tao (2016)

www.caister.com/flu3

- MALDI-TOF Mass Spectrometry in Microbiology Edited by: M Kostrzewa, S Schubert (2016)

www.caister.com/malditof

- Aspergillus and Penicillium in the Post-genomic Era Edited by: RP Vries, IB Gelber, MR Andersen (2016)

"new and well-presented book" (IMA Fungus)

www.caister.com/aspergillus2

- The Bacteriocins: Current Knowledge and Future

Prospects

Edited by: RL Dorit, SM Roy, MA Riley (2016)

www.caister.com/bacteriocins

- Omics in Plant Disease Resistance

Edited by: V Bhadauria (2016)

"essential reading ... highly recommended" (Biotechnol. Agron.

Soc. Environ.)

www.caister.com/opdr

- Acidophiles: Life in Extremely Acidic Environments

Edited by: R Quatrini, DB Johnson (2016)

"Contributors from a wide range of biological and environmental sciences" (ProtoView)

www.caister.com/acidophiles
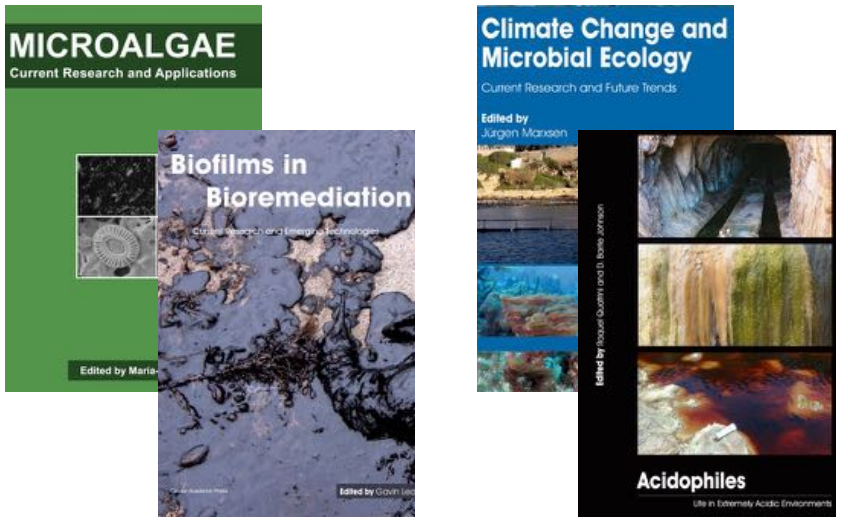
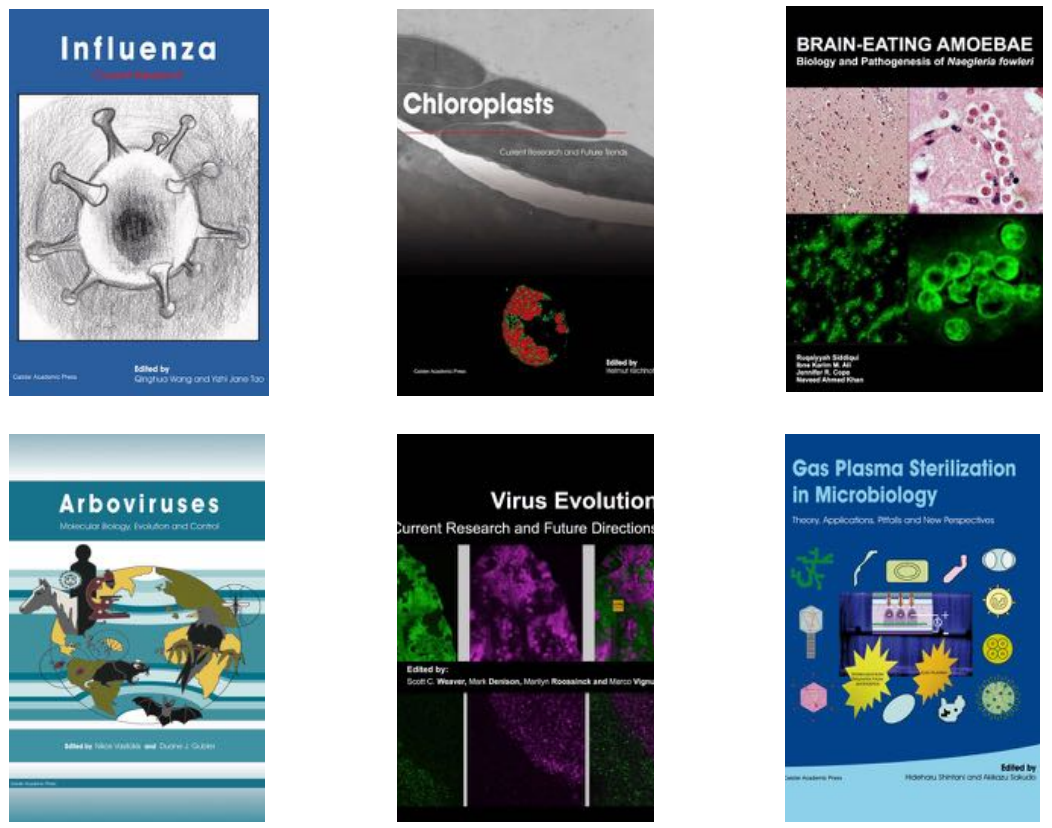

- Climate Change and Microbial Ecology: Current Research and Future Trends

Edited by: J Marxsen (2016)

"impressive" (ASM: Small Things Considered); "written at a high scientific level" (BioSpektrum)

www.caister.com/climate

- Biofilms in Bioremediation: Current Research and Emerging Technologies

Edited by: G Lear (2016)

"describes explicitly the role of biofilms in bioremediation" (Biospektrum); indispensable ... recommended (Biotechnol. Agron. Soc. Environ.) www.caister.com/biorem

- Microalgae: Current Research and Applications

Edited by: MN Tsaloglou (2016)

www.caister.com/microalgae

- Gas Plasma Sterilization in Microbiology: Theory, Applications, Pitfalls and New Perspectives

Edited by: H Shintani, A Sakudo (2016)

"a nice state of the art compilation" (Doodys)

www.caister.com/gasplasma

- Virus Evolution: Current Research and Future Directions Edited by: SC Weaver, M Denison, M Roossinck, et al. (2016) "highly informative ... a pleasure to read" (Microbiol. Today) www.caister.com/virusevol

- Arboviruses: Molecular Biology, Evolution and Control Edited by: N Vasilakis, DJ Gubler (2016)

"a thorough and compelling review ... an outstanding book ... highly recommended" (Am. J. Trop. Med. Hyg.) www.caister.com/arbo

- Shigella: Molecular and Cellular Biology

Edited by: WD Picking, WL Picking (2016)

www.caister.com/shigella

- Aquatic Biofilms: Ecology, Water Quality and Wastewater Treatment

Edited by: AM Romaní, H Guasch, MD Balaguer (2016)

"essential reference book" (Biotechnol. Agron. Soc. Environ.)

www.caister.com/aquaticbiofilms

- Alphaviruses: Current Biology

Edited by: S Mahalingam, L Herrero, B Herring (2016)

"up-to-date review of the field" (Aus. Vet. J.)

www.caister.com/alpha 